\title{
Premorbid Use of Clopidogrel Portends Worse Outcomes in Patients Treated Surgically for Intracranial Hemorrhage
}

\author{
Eric Marvin, Aaron Danison, Greg Davis, Zev Elias, Gary Simonds \\ Virginia Tech Carilion Clinic, Virginia Tech Carilion School of Medicine, Roanoke, Virginia, USA \\ Email: marvin.eric@gmail.com
}

Received 5 July 2014; revised 5 August 2014; accepted 5 September 2014

Copyright (C) 2014 by authors and Scientific Research Publishing Inc.

This work is licensed under the Creative Commons Attribution International License (CC BY). http://creativecommons.org/licenses/by/4.0/

CC) (i) Open Access

\section{Abstract}

Background: Widespread use of antiplatelet and anticoagulation medications (APACs) can be a difficult challenge in the presence of a neurosurgical emergency. Premorbid use of APACs, particularly clopidogrel, has been shown to affect outcomes in patients with stroke and traumatic brain injury. Objective: We hypothesized that pre-morbid clopidogrel use in patients with intracranial hemorrhage necessitating surgical intervention would lead to a greater risk of death and need for re-operation than those taking other APACs. Methods: Retrospective single institution review was conducted from January, 2010 through November, 2012 for intracranial hemorrhages necessitating surgical evacuation. Acute, subacute and chronic subdural, epidural and intraparenchymal hemorrhages were included. Results: 185 of 410 patients that required surgery for intracranial hemorrhage were on APACs. Overall mortality rate was $33 \%$, with a $37 \%$ mortality rate in the APAC group. Overall reoperation rate was $7.5 \%$, and $13 \%$ in the APAC group. Chi-square testing demonstrated significance between mortality and clopidogrel use $(p=0.0038)$, but not in APAC, warfarin or aspirin groups. There was statistical significance between the need for reoperation and APAC use $(p=0.002)$, aspirin use $(p=0.0097)$, and clopidogrel use $(p=0.0152)$, but not warfarin. Multivariate regression demonstrated only clopidogrel use is associated with higher mortality $(p=0.05)$ and need for reoperation $(p=0.0206)$. Conclusion: APAC use in the setting of intracranial hemorrhage necessitating surgical evacuation have higher intraoperative blood loss, need for transfusion and risk for adverse cardiac events. Premorbid clopidogrel use is associated with an increased risk in mortality and need for reoperation.

\section{Keywords}

Antiplatelets, Anticoagulation, Clopidgrel, Mortality, Outcomes, Reoperation 


\section{Introduction}

Patient use of antiplatelet and anticoagulation medications (APACs) has steadily increased over the past two decades [1]-[5]. These medications are prescribed for a variety of medical disorders and conditions and have a proven track record in the treatment of such diseases. However, the premorbid use of APACs has proven to be a difficult challenge in the presence of a neurosurgical emergency. They pose a risk factor for intracranial hemorrhage [1] and have been associated with poor prognoses in the settings of both spontaneous as well as trauma-related hemorrhage [6]-[10]. With respect to spontaneous hemorrhage, antithrombotic therapy is associated with 7000 of the 60,000 (12\%) intracerebral hemorrhages (ICH) that occur annually in the United States (which represents approximately $1 \%$ of incidental strokes) [11]. In the setting of trauma, APACs contribute to both the incidence as well as the severity of accompanying intracranial hemorrhage [10] [12]-[14]. Warfarin use may double the attendant risk of ICH. Risk increases exponentially as the INR rises beyond recommended therapeutic levels. In the general population, aspirin use may increase the risk of ICH by as much as $40 \%$ [11]. In addition, it is not uncommon for patients to be placed on a dual or even a triple regimen of APAC medications. The combined use of two or more medications appears to carry a greater risk of spontaneous ICH than does the use of single agents [11] [15]-[17]. Given the increasing use of APACs [1]-[5] and the rapid development of newer agents, the neurosurgical implications are significant.

Neurosurgical intervention for intracranial hemorrhage is dependent on a number of factors including the hematoma size, mass effect, and neurological status of the patient. However, the initiation of surgical evacuation also involves consideration of the natural history of intracranial hemorrhage, and an understanding of the patient's overall prognosis. Regarding ICH, recent investigations have addressed issues such as risk for hematoma expansion [13] [18]-[20], outcomes of intracranial hemorrhage in patients taking APACs [6]-[10] and differences in said outcomes between the various types of antithrombotics [10] [21]-[25]. These studies were performed primarily with regard to stroke, emergent care and care in a trauma setting, not to neurosurgical intervention. To our knowledge, there are no studies specifically addressing the surgical outcomes of this patient population. Furthermore, we have not identified any specific studies that differentiate surgical outcomes between patients pre-morbidly taking antiplatelet medications and those taking anticoagulant medications. Our experience with intracranial hemorrhage suggested that outcomes for patients taking clopidogrel were worse than those in patients taking warfarin. We hypothesized that in patients suffering from an intracranial hemorrhage necessitating surgical intervention, pre-morbid clopidogrel use would place patients at a greater risk of death and need for re-operation than those taking other APACs. We sought to test this hypothesis by reviewing our outcomes for patients treated at our institution for intracranial hemorrhage over a 2-year period. We believe that these results have potentially significant implications with respect to the consideration of treatment options, patient and family counseling, and directions for future investigation (Figure 1).

\section{Materials and Methods}

An IRB approved, single institution, retrospective Chart 1 review was conducted on surgically treated intracranial hemorrhage patients at Virginia Tech Carilion from January, 2010, through November, 2012. Chart 2 reviews were conducted through the institutions electronic medical record (EPIC). Surgical hemorrhages included acute, subacute, and chronic subdural, epidural, and intraparenchymal hemorrhages. Aneurysms necessitating craniotomy for clot evacuation were included, but surgeries for elective aneurysm clipping related to aneurysmal subarachnoid hemorrhage were not. Patients initially undergoing surgery at outside institutions were excluded.

Patient information collected included medical record number, date of surgery, time of surgery, the surgeon performing the procedure, sex, date of birth, age of the patient, date of first surgery, date of second surgery (if performed), the type of surgery performed, the type and location of hemorrhage, whether any reversal products were given (platelets, ddAVP, FFP, factor VII, vitamin K, PCC, etc.), whether blood transfusions were given, presence of cardiac events, whether the patient was on an APAC (and if so which medication), presenting INR and last INR prior to surgery, whether the pathology was trauma related, the imaging characteristics including width/length/height/volume and midline shift, Glasgow Coma Scores (GCS) and GCS motor scores, anisocoria, medical comorbidities, ETOH or illicit drug use, height/weight and BMI of patient.

The study group consisted of all patients undergoing surgical evacuation for intracranial hemorrhage, both spontaneous and traumatic. The primary outcomes analyzed were mortality and need for re-operation. An analysis was performed comparing all patients taking APACs with those who were not. In addition, specific medica- 


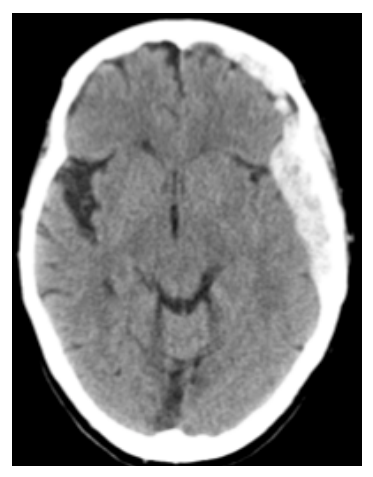

(a)

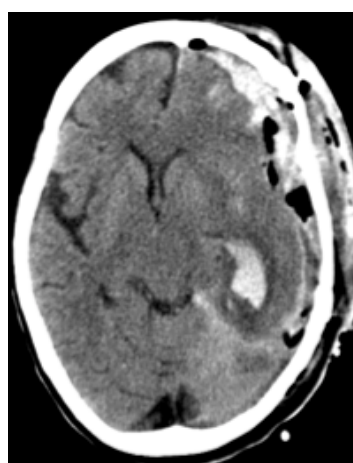

(b)

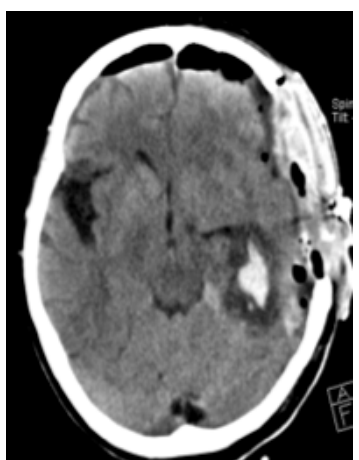

(c)

Figure 1. (a) Moderate left sided acute SDH with mild mass effect and deteriorating neurological exam in a patient taking clopidogrel-patient was taken for emergent craniotomy; (b) Recurrent $\mathrm{SDH}$, now with an acute left posterior temporal ICH and increased midline shift (MLS) necessitating second surgery; (c) Improved MLS after craniectomy and decompression.

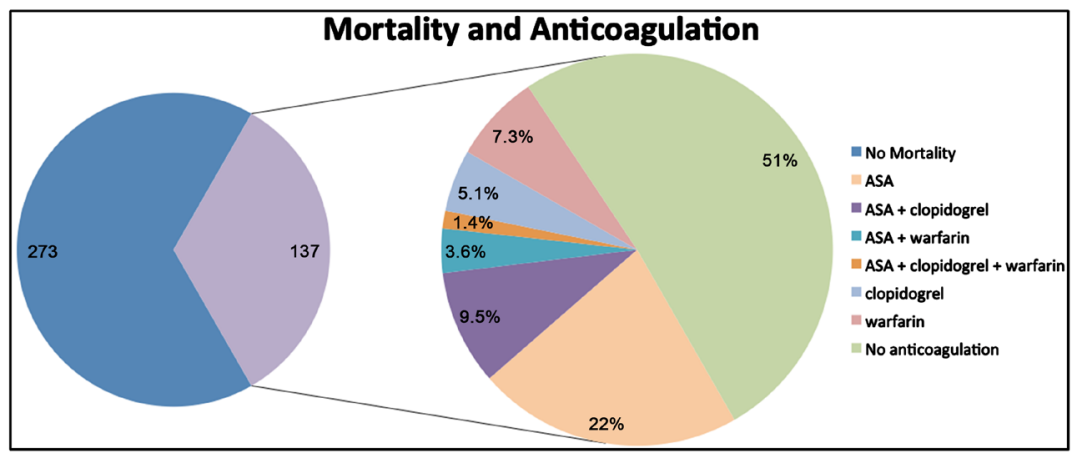

Chart 1. Relationship between anticoagulation/antiplatelet use/nonuse and mortality.

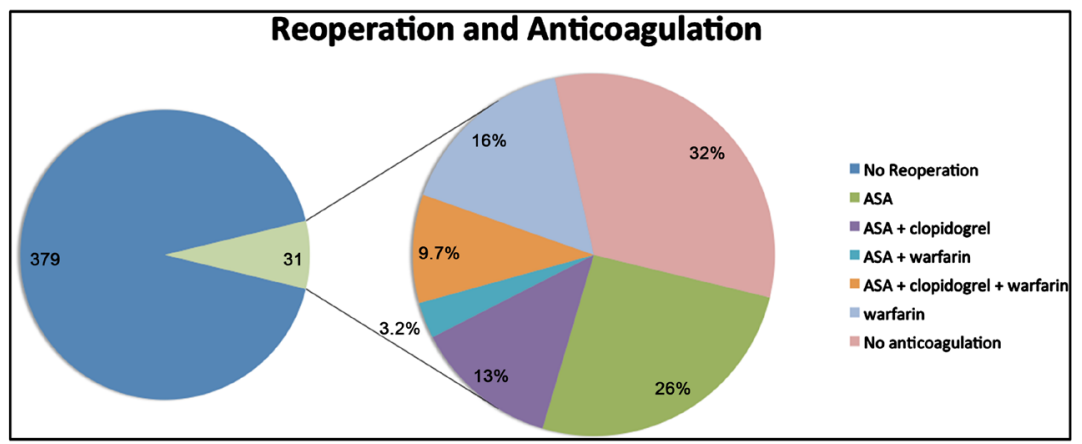

Chart 2. Relationship between anticoagulation/antiplatelet use/nonuse and need for reoperation.

tion subgroups were compared. For statistical power, we included only comparisons between groupings of warfarin, clopidogrel and aspirin. Secondary outcomes evaluated included total hospital days, EBL, blood transfusions required, cardiac events, ventilator days and ICU days.

Statistical analysis was performed using Chi-square contingency testing and multivariate regression techniques utilizing the FREQ procedure, T-Test comparison and LOGISTIC analysis.

\section{Results}

410 patients underwent surgery for intracranial hemorrhage between January, 2010, and November, 2012, for intracranial hemorrhages. 310 were trauma related. The average age of patients in this study was 60.1 years with 
$69 \%$ being males. The average presenting GCS was 10.5 .

185 (45\%) patients were premorbidly taking APACs. 127 patients were on aspirin, 41 were on clopidogrel and 58 were on warfarin. 12 patients had received enoxaparin, 4 were on heparin, 3 patients had received tissue plasminogen activator, 2 patients were on Aggrenox, 2 patients had received prasugrel, 2 patients had received eptifibatide, 1 patient was on dabigatran, 1 patient was on cilostazol. Of patients treated with clopidogrel, 32 were also on aspirin, 3 were on enoxaparin, 3 were on warfarin, and 4 were on other APACs. In the warfarin group, 16 were on aspirin. The average INR of patients presenting on warfarin was 2.3 (range 1.2 to 8).

A total of 137 patients died (33\% of total) within 90 days of presentation. 69 of the patients who died were on an APAC (37\% mortality rate overall in this group) and 68 patients were not (30\% mortality rate overall in this group). 31 patients overall required additional surgery, 24 of which were on an APAC (77\%). Of patients that required reoperation, 16 died (52\%). 15 of the 16 patients who died after reoperation were taking an APAC.

The average GCS for patients presenting on an APAC was 11.6 compared with 9.8 for patients who were not on an APAC. The average estimated blood loss for the APAC group was $195.5 \mathrm{~mL}$ during surgery vs. $187.2 \mathrm{~mL}$. There were 41 packed red blood cell transfusions (PRBC) for the APAC group (24\%) vs. 49 for the non-APAC group (21\%). There were 65 cardiac events that occurred in the APAC group (35\%) vs. 20 in the non APAC group (9\%); these included documented new onset bradycardia, left bundle branch blocks, arrhythmias including atrial fibrillation with and without rapid ventricular rate, supraventricular and ventricular tachycardia, myocardial infarction, non-ST elevated myocardial infarction, congestive heart failure, pulseless electrical activity and cardiac related hypotension. The average ventilator days for patients taking an APAC were 6, ranging from 0 to 53 compared to 7 days for patients not on an APAC. The average ICU stay was 6 days for the APAC group and 7 for the non-APAC group.

Subgroup analysis for the aspirin groups (127 patients) demonstrated 50 deaths (39\%) and 16 patients that required reoperation (11\%). 93 were trauma related. The average GCS was 11.6. The average EBL was $206 \mathrm{~mL}$ and 33 patients required blood transfusions (26\%). There were 42 cardiac events (33\%). The average ventilator days were 6 and an average ICU stay of 7 days.

For the clopidogrel group (41 patients), there were 22 deaths (54\%) and 7 patients required reoperation (17\%). 29 were trauma related. The average GCS was 10.8. The average EBL was $255 \mathrm{~mL}$ and 17 patients required blood transfusions (41\%). There were 22 cardiac events (54\%). The average ventilator days were 7 and an average ICU stay of 7 days. In patients on clopidogrel, not receiving aspirin (9), there was one patient that needed a repeat operation and 7 mortalities.

For the warfarin group (58 patients), there were 17 deaths (29\%) and 7 patients required reoperation (12\%). 44 were trauma related (76\%). The average GCS was 12. The average EBL was 195 cc and 11 patients required blood transfusions (19\%). There were 24 cardiac events (41\%). The average ventilator days were 4 and an average ICU stay of 5 days.

Pearson's Chi-square contingency testing with 95\% confidence interval was used for analysis of primary and secondary outcomes. With respect to mortality, our study found that there was no significant association between APAC use in general and mortality $(\mathrm{p}=0.13)$, warfarin and mortality $(\mathrm{p}=0.47)$, aspirin and mortality ( $\mathrm{p}$ $=0.09)$ and not being on an APAC and mortality $(\mathrm{p}=0.13)$. In addition, there was no statistically significant association between reoperation and warfarin use $(\mathrm{p}=0.16)$.

With respect to primary outcomes however, Chi Square testing did show statistical significance between APAC use and need for reoperation ( $<<0.001)$, aspirin use and the need for reoperation $(p=0.010)$, clopidogrel use and mortality $(p=0.004)$ and clopidogrel use and need for reoperation $(p=0.015)$.

As a measure of secondary outcomes, there was no statistically significant association between the use of an APAC, aspirin, or warfarin and the need for PRBC Transfusion ( $\mathrm{p}=0.93,0.12,0.55$ respectively). The use of clopidogrel and the need for transfusion was found to be significant with a p-value of 0.001 . Aspirin, warfarin, clopidogrel and APAC as a whole were all associated with adverse cardiac events ( $\mathrm{p}<0.001$ for each measure). In comparison of continuous variables, ICU level of stay and ventilator days were both associated with increased mortality ( $\mathrm{p}<0.001$ for each).

Because of the significant confounding variables that we felt were inherent in this study; age, the presence of trauma, and the presenting GCS score, were accounted for statistically by using multivariate logistic regression models. Tests of association were made for gender, age, age groups, trauma and GCS scores. When adjusted for baseline/demographic variables, trauma, GCS score, and age were associated with higher mortality and found to be statistically significant when considered univariately. GCS score when considered univariately, was statistically associated with need for reoperation. 
Multivariate logistic regression showed that when adjusting for trauma, GCS score and age, APAC use as a whole was not associated with higher mortality. However, when adjusted for GCS score, APAC use, and the use of clopidogrel ( $\mathrm{Pr}>$ ChiSq 0.021, OR 2.977 95\% CI, 1.182 - 7.500), is associated significantly with the need for second surgery. When individual APAC use is adjusted for trauma, GCS score and age, only clopidogrel is significantly associated with higher mortality $(\mathrm{p}=0.05)$. There was not enough evidence to detect an association between aspirin or warfarin and increased mortality or need for reoperation.

\section{Discussion}

The pre-morbid use of APAC medications in the setting of intracranial hemorrhage requiring surgical evacuation has an adverse impact on outcomes. In addition, the impact on outcomes appears to vary with the type of APAC used.

Patients with surgical intracranial hemorrhages require aggressive perioperative management particularly with respect to the reversal of anti-coagulation and/or ant-platelets as well as strict blood pressure control. We had suspected from anecdotal experiences that patients taking clopidogrel pre-morbidly faired worse than those taking other APACs, perhaps due to the irreversibility of the agent in the acute setting (unlike warfarin for example). In this study we confirmed that patients taking clopidogrel required a greater number of re-operations for re-hemorrhage, and suffered a greater mortality rate than did patients taking other APACs (and patients taking no APACs). These findings may need to be considered and discussed when considering surgery for an intracranial hemorrhage in a patient taking clopidogrel.

Irreversible APACs are empirically problematic in the realm of urgent and emergent neurosurgical interventions. In our study, although patients on warfarin faired more poorly than patients not taking APACS in several outcome measures, they demonstrated better outcomes than did the patients taking clopidogrel. In our institution, patients with a significant intracranial hemorrhage who are taking warfarin are reversed effectively and rapidly with combinations of prothrombin complex concentrate (PCC), fresh frozen plasma (FFP), and Vitamin K. Although multiple regimens have been suggested and employed in patients taking clopidogrel (currently platelet transfusion and DDAVP), no definitive reversal regimen exists. Thus procedures are performed, and post-operative care is affected, against the backdrop of continued platelet inactivation. Not surprisingly, our study confirms that these patients fair worse than those taking no APACs in multiple outcome categories such as mortality, need for repeat surgery, EBL, need for transfusions, and adverse cardiac events. Aspirin, another irreversible platelet inhibitor, was also associated with worse outcomes.

The difficulties in achieving reversibility of antiplatelet medications in an emergent surgical scenario highlight the need for better treatment protocols. In addition, future interventions for intracranial hemorrhage can be anticipated to become increasingly challenging as the market becomes saturated with new, irreversible APACs such as dabigatran, rivaroxaban and apixaban. Such agents have the allure of requiring no coagulation-profile monitoring, but possess no effective antidote and/or universally accepted reversal protocol. This consideration is hardly featured prominently in "direct-to-the customer" advertising readily seen in multi-media campaigns. Strikingly, most hospital pharmacies have no suggested reversal protocols in place for many of these agents.

Potential confounders of this study include the comorbidities that often accompany the use of anticoagulation and antiplatelet agents. Statistical methodology helps to minimize these confounders to some extent, however the age difference between study groups was definitive: non-APAC age group averaged 50.6 years, compared to the APAC group of 71.7 years. In addition, many of the patients on clopidogrel were on more than one agent, which is a known risk factor for ICH [11] [15]-[17]. There were only 9 patients taking clopidogrel as a single agent, making statistical analysis underpowered when looking at clopidogrel alone; thus the results of this study may be best looked at in terms of dual antiplatelet therapy. Though not measured directly in this study, we acknowledge that the number and severity of comorbidities of patients taking APAC medications are likely to be significantly increased when compared to those who are not taking such medications; the differences of comorbidities among the varying APAC subgroups is less clear.

A key issue is the optimal treatment strategy of intracranial hemorrhage in the face of clopidogrel use. The literature would suggest that there is no definitive reversal algorithm that has been established in the treatment of antiplatelet related intracranial hemorrhage [15] [26]-[28], but aggressive platelet transfusion empirically seems appropriate in this context. Ideally, outcomes in such patients with varying reversal protocols would be studied.

Another phenomenon to be mentioned in a post-hoc review was a pattern of malignant edema seen post-operatively in several patients taking clopidogrel (see Figure 2). When this pattern occurred, we were often forced 


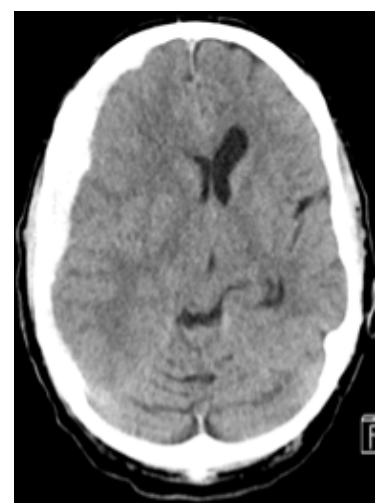

(a)

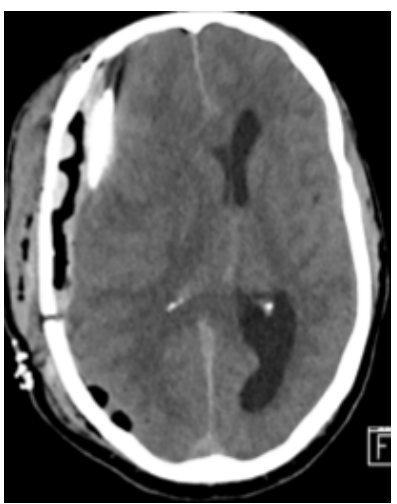

(b)

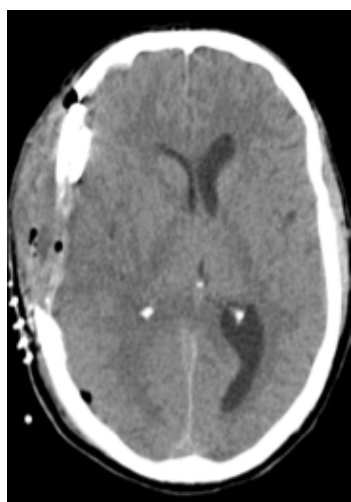

(c)

Figure 2. (a) Moderately acute right sided SDH with significant midline shift; (b) Craniotomy with evacuation of hemorrhage and development of increasing midline shift, with neurological deterioration; (c) Subsequent decompressive craniectomy and improvement in midline shift.

to return to the operating room for full decompressive craniectomies. We are not aware of descriptions of similar phenomena in this context and are not aware of a pathophysiological mechanism behind it.

It is unknown to what extent practitioners who prescribe APACs are aware of the medications' potential dire neurological consequences, and the neurosurgical challenges they may present. We were not able to ascertain data on the general use of APACs in our geographical region, though our study identified that of the 410 patients requiring surgical evacuation for intracranial hemorrhage over the course of 23 months, 185 were on some form of an APAC. It has been suggested that an increase of $1 \%-2 \%$ of ICH rates can negate the benefits of antithrombotic therapy [11]. It should be noted that while the mortality of our study is disproportionately higher to what has been described in the literature [29] [30], this is the first paper, to our knowledge, that assesses the adverse effects of APAC use on patients presenting with surgically-treated intracranial hemorrhage.

\section{Conclusion}

Patients pre-morbidly taking APACs suffer worse outcomes than those who are not, when acute intracranial hemorrhage necessitates surgical evacuation. Those taking clopidogrel fair worse than patients on other APACs with a statistically significant increase in mortality and need for repeat operations. Increased adverse outcomes need to be acknowledged when considering surgical intervention for patients with intracranial hemorrhage taking APACS, particularly clopidogrel. Ideally, acute reversal agents should be available for all APACs. In short of this, reversal or mitigating-agent protocols should be investigated and disseminated for each of the APAC agents.

\section{Conflicts of Interest}

All authors declare there are no conflicts of interest.

\section{References}

[1] Dossett, L.A., Riesel, J.N., Griffin, M.R. and Cotton, B.A. (2011) Prevalence and Implications of Preinjury Warfarin Use: An Analysis of the National Trauma Databank. Archives of Surgery, 146, 565-570. http://dx.doi.org/10.1001/archsurg.2010.313

[2] IMS Institute for Healthcare Informatics (2010) The Use of Medicines in the United States: Patient Payment for Medicines.

http://www.imshealth.com/deployedfiles/imshealth/Global/Content/IMS\%20Institute/Static\%20File/IHII_UseOfMed_r eport.pdf

[3] Ostini, R., Hegney, D., Mackson, J.M., Williamson, M. and Tett, S.E. (2008) Why Is the Use of Clopidogrel Increasing Rapidly in Australia? An Exploration of Geographical Location, Age, Sex and Cardiac Stenting Rates as Possible Influences on Clopidogrel Use. Pharmacoepidemiol Drug Safety, 17, 1077-1090. http://dx.doi.org/10.1002/pds.1638

[4] Rao, R.V., Goodman, S.G., Yan, R.T., Spencer, F.A., Fox, K.A., DeYoung, J.P., et al. (2009) Temporal Trends and 
Patterns of Early Clopidogrel Use across the Spectrum of Acute Coronary Syndromes. American Heart Journal, 157, 642-650 e641. http://dx.doi.org/10.1016/j.ahj.2009.01.002

[5] Virjo, I., Makela, K., Aho, J., Kalliola, P., Kurunmaki, H., Uusitalo, L., et al. (2010) Who Receives Anticoagulant Treatment with Warfarin and Why? A Population-Based Study in Finland. Scandinavian Journal of Primary Health Care, 28, 237-241. http://dx.doi.org/10.3109/02813432.2010.514138

[6] Joseph, B., Pandit, V., Aziz, H., Kulvatunyou, N., Hashmi, A., Tang, A., et al. (2014) Clinical Outcomes in Traumatic Brain Injury Patients on Preinjury Clopidogrel: A Prospective Analysis. Journal of Trauma and Acute Care Surgery, 76, 817-820. http://dx.doi.org/10.1097/ta.0b013e3182aafcf0

[7] Nishijima, D.K., Offerman, S.R., Ballard, D.W., Vinson, D.R., Chettipally, U.K., Rauchwerger, A.S., et al. (2013) Risk of Traumatic Intracranial Hemorrhage in Patients with Head Injury and Preinjury Warfarin or Clopidogrel Use. Academic Emergency Medicine, 20, 140-145. http://dx.doi.org/10.1111/acem.12074

[8] Roquer, J., Rodriguez Campello, A., Gomis, M., Ois, A., Puente, V. and Munteis, E. (2005) Previous Antiplatelet Therapy Is an Independent Predictor of 30-Day Mortality after Spontaneous Supratentorial Intracerebral Hemorrhage. Journal of Neurology, 252, 412-416. http://dx.doi.org/10.1007/s00415-005-0659-5

[9] Saloheimo, P., Ahonen, M., Juvela, S., Pyhtinen, J., Savolainen, E.R. and Hillbom, M. (2006) Regular Aspirin-Use Preceding the Onset of Primary Intracerebral Hemorrhage Is an Independent Predictor for Death. Stroke, 37, 129-133. http://dx.doi.org/10.1161/01.str.0000196991.03618.31

[10] Peck, K.A., Calvo, R.Y., Schechter, M.S., Sise, C.B., Kahl, J.E., Shackford, M.C., et al. (2014) The Impact of Preinjury Anticoagulants and Prescription Antiplatelet Agents on Outcomes in Older Patients with Traumatic Brain Injury. Journal of Trauma and Acute Care Surgery, 76, 431-436. http://dx.doi.org/10.1097/ta.0000000000000107

[11] Hart, R.G., Tonarelli, S.B. and Pearce, L.A. (2005) Avoiding Central Nervous System Bleeding during Antithrombotic Therapy: Recent Data and Ideas. Stroke, 36, 1588-1593. http://dx.doi.org/10.1161/01.str.0000170642.39876.f2

[12] Fabbri, A., Servadei, F., Marchesini, G., Bronzoni, C., Montesi, D., Arietta, L., et al. (2013) Antiplatelet Therapy and the Outcome of Subjects with Intracranial Injury: The Italian SIMEU Study. Critical Care, 17, R53. http://dx.doi.org/10.1186/cc12575

[13] Nishijima, D.K., Offerman, S.R., Ballard, D.W., Vinson, D.R., Chettipally, U.K., Rauchwerger, A.S., et al. (2012) Immediate and Delayed Traumatic Intracranial Hemorrhage in Patients with Head Trauma and Preinjury Warfarin or Clopidogrel Use. Annals of Emergency Medicine, 59, 460-468. http://dx.doi.org/10.1016/j.annemergmed.2012.04.007

[14] Nishijima, D.K., Shahlaie, K., Sarkar, K., Rudisill, N. and Holmes, J.F. (2013) Risk of Unfavorable Long-Term Outcome in Older Adults with Traumatic Intracranial Hemorrhage and Anticoagulant or Antiplatelet Use. The American Journal of Emergency Medicine, 31, 1244-1247. http://dx.doi.org/10.1016/j.ajem.2013.04.035

[15] Campbell, P.G., Sen, A., Yadla, S., Jabbour, P. and Jallo, J. (2010) Emergency Reversal of Antiplatelet Agents in Patients Presenting with an Intracranial Hemorrhage: A Clinical Review. World Neurosurgery, 74, 279-285. http://dx.doi.org/10.1016/j.wneu.2010.05.030

[16] Diener, H.C., Bogousslavsky, J., Brass, L.M., Cimminiello, C., Csiba, L., Kaste, M., et al. (2004) Aspirin and Clopidogrel Compared with Clopidogrel Alone after Recent Ischaemic Stroke or Transient Ischaemic Attack in High-Risk Patients (MATCH): Randomised, Double-Blind, Placebo-Controlled Trial. The Lancet, 364, 331-337. http://dx.doi.org/10.1016/s0140-6736(04)16721-4

[17] Hart, R.G., Benavente, O. and Pearce, L.A. (1999) Increased Risk of Intracranial Hemorrhage When Aspirin Is Combined with Warfarin: A Meta-Analysis and Hypothesis. Cerebrovascular Diseases, 9, 215-217. http://dx.doi.org/10.1159/000015958

[18] Dowlatshahi, D., Butcher, K.S., Asdaghi, N., Nahirniak, S., Bernbaum, M.L., Giulivi, A., et al. (2012) Poor Prognosis in Warfarin-Associated Intracranial Hemorrhage Despite Anticoagulation Reversal. Stroke, 43, 1812-1817. http://dx.doi.org/10.1161/strokeaha.112.652065

[19] Joseph, B., Sadoun, M., Aziz, H., Tang, A., Wynne, J.L., Pandit, V., et al. (2014) Repeat Head Computed Tomography in Anticoagulated Traumatic Brain Injury Patients: Still Warranted. The American Journal of Surgery, 80, 43-47.

[20] Levine, M., Wyler, B., LoVecchio, F., Roque, P. and Raja, A.S. (2014) Risk of Intracranial Injury after Minor Head Trauma in Patients with Pre-Injury Use of Clopidogrel. The American Journal of Emergency Medicine, 32, 71-74. http://dx.doi.org/10.1016/j.ajem.2013.08.063

[21] Bonville, D.J., Ata, A., Jahraus, C.B., Arnold-Lloyd, T., Salem, L., Rosati, C., et al. (2011) Impact of Preinjury Warfarin and Antiplatelet Agents on Outcomes of Trauma Patients. Surgery, 150, 861-868. http://dx.doi.org/10.1016/j.surg.2011.07.070

[22] Falzon, C.M., Celenza, A., Chen, W. and Lee, G. (2013) Comparison of Outcomes in Patients with Head Trauma, Taking Preinjury Antithrombotic Agents. Emergency Medicine Journal, 30, 809-814. http://dx.doi.org/10.1136/emermed-2012-201687 
[23] He, J., Whelton, P.K., Vu, B. and Klag, M.J. (1998) Aspirin and Risk of Hemorrhagic Stroke: A Meta-Analysis of Randomized Controlled Trials. JAMA, 280, 1930-1935. http://dx.doi.org/10.1001/jama.280.22.1930

[24] Mina, A.A., Knipfer, J.F., Park, D.Y., Bair, H.A., Howells, G.A. and Bendick, P.J. (2002) Intracranial Complications of Preinjury Anticoagulation in Trauma Patients with Head Injury. Journal of Trauma-Injury Infection \& Critical Care, 53, 668-672. http://dx.doi.org/10.1097/00005373-200210000-00008

[25] Rosand, J., Eckman, M.H., Knudsen, K.A., Singer, D.E. and Greenberg, S.M. (2004) The Effect of Warfarin and Intensity of Anticoagulation on Outcome of Intracerebral Hemorrhage. Archives of Internal Medicine, 164, 880-884. http://dx.doi.org/10.1001/archinte.164.8.880

[26] Altman, R., Scazziota, A., Herrera, M. and Gonzalez, C. (2006) Recombinant Factor VIIa Reverses the Inhibitory Effect of Aspirin or Aspirin plus Clopidogrel on in Vitro Thrombin Generation. Journal of Thrombosis and Haemostasis, 4, 2022-2027.

[27] Beshay, J.E., Morgan, H., Madden, C., Yu, W. and Sarode, R. (2010) Emergency Reversal of Anticoagulation and Antiplatelet Therapies in Neurosurgical Patients. Journal of Neurosurgery, 112, 307-318. http://dx.doi.org/10.3171/2009.7.jns0982

[28] Gordon, J.L., Fabian, T.C., Lee, M.D. and Dugdale, M. (2013) Anticoagulant and Antiplatelet Medications Encountered in Emergency Surgery Patients: A Review of Reversal Strategies. Journal of Trauma and Acute Care Surgery, 75, 475-486. http://dx.doi.org/10.1097/ta.0b013e3182a07391

[29] Ohm, C., Mina, A., Howells, G., Bair, H. and Bendick, P. (2005) Effects of Antiplatelet Agents on Outcomes for Elderly Patients with Traumatic Intracranial Hemorrhage. Journal of Trauma-Injury Infection \& Critical Care, 58, 518522. http://dx.doi.org/10.1097/01.ta.0000151671.35280.8b

[30] Peck, K.A., Calvo, R.Y., Schechter, M.S., Sise, C.B., Kahl, J.E., Shackford, M.C., et al. (2014) The Impact of Preinjury Anticoagulants and Prescription Antiplatelet Agents on Outcomes in Older Patients with Traumatic Brain Injury. Journal of Trauma and Acute Care Surgery, 76, 431-436. http://dx.doi.org/10.1097/ta.0000000000000107 
Scientific Research Publishing (SCIRP) is one of the largest Open Access journal publishers. It is currently publishing more than 200 open access, online, peer-reviewed journals covering a wide range of academic disciplines. SCIRP serves the worldwide academic communities and contributes to the progress and application of science with its publication.

Other selected journals from SCIRP are listed as below. Submit your manuscript to us via either submit@scirp.org or Online Submission Portal.
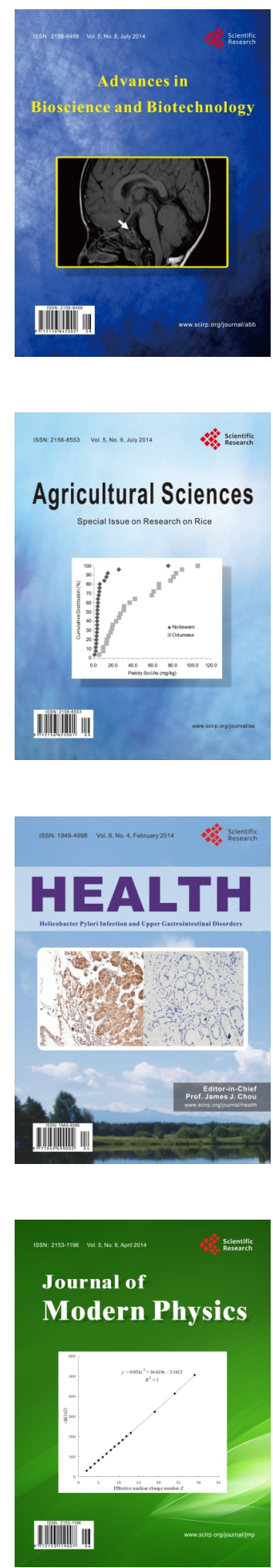
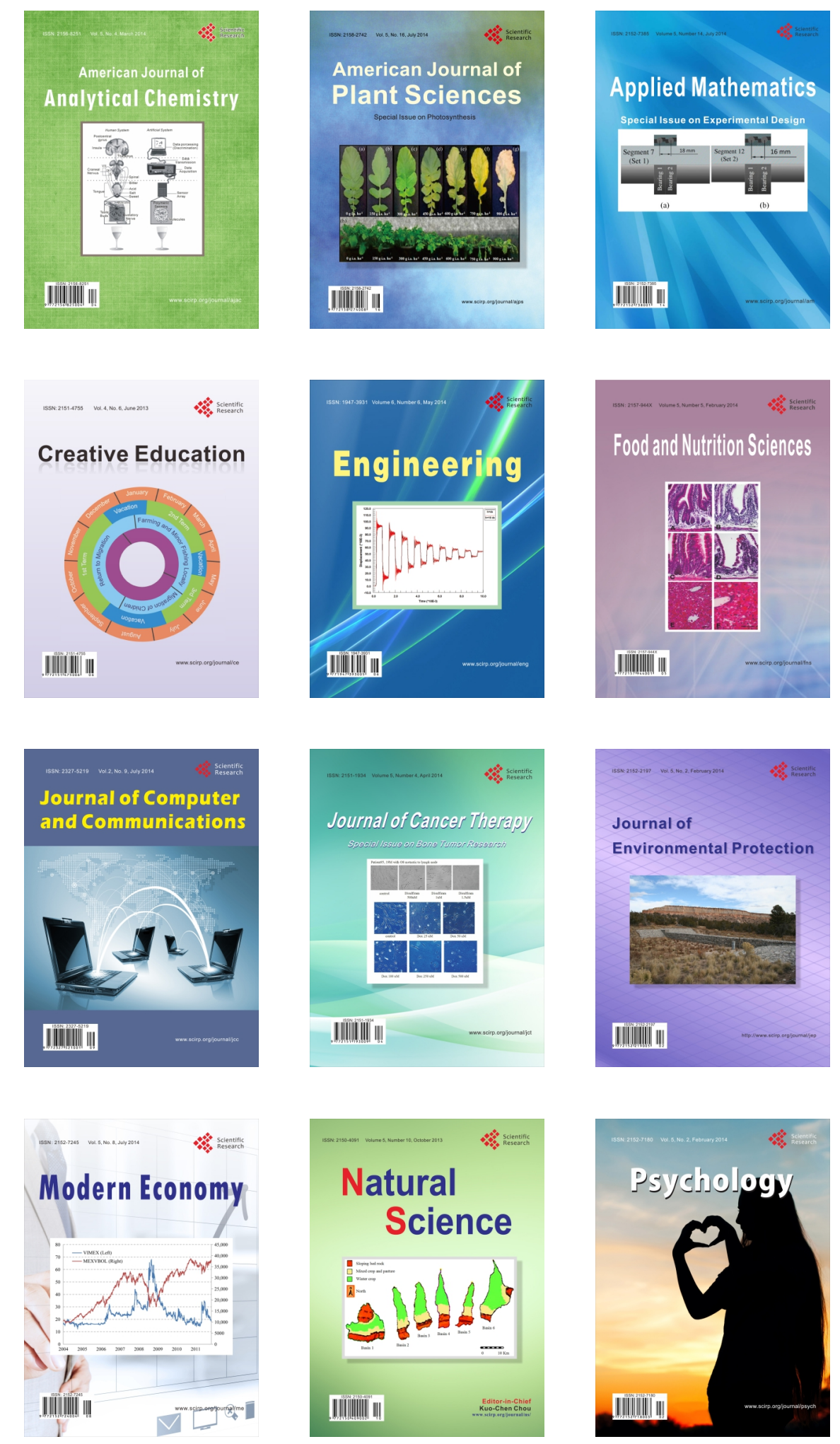